International Journal of Pure and Applied Mathematics

Volume 85 No. $6 \quad 2013,1065-1073$

ISSN: $1311-8080$ (printed version); ISSN: 1314-3395 (on-line version)

url: http://www.ijpam.eu

doi: http://dx.doi.org/10.12732/ijpam.v85i6.10

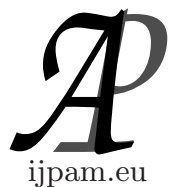

\title{
UNION OF LINES IN PROJECTIVE SPACES AND THEIR INTERSECTION WITH QUADRICS AND HYPERPLANES
}

\author{
E. Ballico \\ Department of Mathematics \\ University of Trento \\ 38123 Povo (Trento) - Via Sommarive, 14, ITALY
}

\begin{abstract}
A tree $Y \subset \mathbb{P}^{r}$ is a connected nodal curve with lines as components and $p_{a}(Y)=0$. Here we investigate the existence of certain trees with nice intersection with a quadric $Q$ or a hyperplane $H$. E.g. we find $Y$ with prescribed $Y \cap H$ or with $h^{1}\left(Q, \mathcal{I}_{Q \cap Y}(t)\right)=0$.
\end{abstract}

AMS Subject Classification: 14H50, 14N05

Key Words: union of lines, postulation, intersection with a quadric

\section{Introduction}

Let $Y \subset \mathbb{P}^{r}$ be a reduced and connected curve. We say that $Y$ is a tree if it is nodal, the irreducible components of $Y$ are lines and $p_{a}(Y)=0$. We look at the intersection of "sufficiently general " trees with hyperplanes and quadric hypersurfaces. To explain the words "sufficiently general " we need to remember that the set of all trees of fixed degree and fixed "topological type " or " combinatorial type" in $\mathbb{P}^{r}, r \geq 3$, is irreducible. We explain it now, calling "type" the topological (or combinatorial) type. A type $\tau$ for an integer $d \geq 2$ is a function $\tau:\{2, \ldots d\} \rightarrow\{1, \ldots, d-1\}$ such that $\tau(i)<i$ for all $i$. Fix a type $\tau$. Let $Z(r, d, \tau)$ be the set of all degree $d$ trees $Y \subset \mathbb{P}^{r}$ such that there is an ordering $L_{i}, 1 \leq i \leq d$, of the irreducible components of $Y$ with $L_{i} \cap L_{\tau(i)} \neq \emptyset$ for all $i=2, \ldots, d$. Let $Z(r, 1, \tau)$ denote the Grassmannian

Received: May 17, 2013

(c) 2013 Academic Publications, Ltd. url: www.acadpubl.eu 
of lines in $\mathbb{P}^{r}$. A tree of degree $d \geq 4$ may have several types, i.e. we may have $Z(r, d, \tau)=Z\left(r, d, \tau^{\prime}\right)$ for some $\tau \neq \tau^{\prime}$. Each set $Z(r, d, \tau)$ is a non-empty irreducible subset of the Hilbert scheme of $\mathbb{P}^{r}$ and it makes sense to speak about the general $Y \in Z(r, d, \tau)$. A tree $Y \subset \mathbb{P}^{r}$ of degree $d \geq 2$ is said to be a comb if the constant function $\eta:\{2, \ldots, d\} \rightarrow\{1\}$ is its type; if $Y=L_{1} \cup \cdots \cup L_{d}$ is an ordering of the lines of $Y$ with $\eta$ as its type, then $L_{i}, 2 \leq i \leq d$, are the tooth of $Y$, while $L_{1}$ is the spine of $Y$. A bamboo of degree $d \geq 2$ is a degree $d$ tree $Y=L_{1} \cup \cdots \cup L_{d}$ which admits the function $i \mapsto i-1$ as a type. Any line will be called a comb and a bamboo of degree 1 .

We will prove the following results, whose proof is a sharp version of a method used in the first version (not the published one) of [1].

Theorem 1. Fix integers $a \geq 0, b \geq 0, d>0$ such that $(a, b, d) \neq(1,1,2)$. Let $Q \subset \mathbb{P}^{3}$ be a smooth quadric surface. Then there is a degree $d$ comb $Y \subset \mathbb{P}^{3}$ intersecting transversally $Q$ and such that either $h^{0}\left(Q, \mathcal{I}_{Y \cap Q}(a, b)\right)=0$ (case $2 d \geq(a+1)(b+1))$ or $h^{1}\left(Q, \mathcal{I}_{Y \cap Q}(a, d)\right)=0$ (case $\left.2 d \leq(a+1)(b+1)\right)$.

Theorem 2. Fix integers $r \geq 3, e \in\{0, \ldots, r\}, d>0$ and $t>0$. Let $Q \subset \mathbb{P}^{r}$ be a rank e quadric hypersurface. Then there is a degree $d$ tree $Y \subset \mathbb{P}^{r}$ such that $Q$ contains no line of $Y, h^{0}\left(Q, \mathcal{I}_{Y \cap Q}(t)\right)=\max \left\{0,\left(\begin{array}{c}r+t \\ r\end{array}\right)-\left(\begin{array}{c}r+t-2 \\ r\end{array}\right)-2 d\right\}$ and $h^{1}\left(Q, \mathcal{I}_{Y \cap Q}(t)\right)=\max \left\{0,2 d-\left(\begin{array}{c}r+t \\ r\end{array}\right)+\left(\begin{array}{c}r+t-2 \\ r\end{array}\right)\right\}$.

As an immediate corollary of Proposition 2 we get the following result.

Corollary 1. Fix a hyperplane $H \subset \mathbb{P}^{r}, r \geq 3$, a finite set $S \subset H$ and $P \in S$. Let $E \subset H$ be the union of all lines containing at least 3 points of $S$. There is a comb $Y \subset \mathbb{P}^{r}$ such that $Y \cap H=S$ if and only if $S \nsubseteq E$.

\section{Proof of Theorems 1 and 2}

Proof of Theorem 1. Fix a line $L_{1} \subset Q$ intersecting transversally $Q$. Notice that the two points of $L_{1} \cap Q$ are not contained in a line of $Q$. In each case we will take as $Y$ a comb with $L_{1}$ as its spine. Write $\left\{O, O^{\prime}\right\}:=L_{1} \cap Q$.

(i) Fix $(a, b)$ with $(a, b) \neq(1,1)$ and assume that we need to prove Proposition 1 for the integer $d$. Set $t:=\lfloor(a+1)(b+1) / 2\rfloor$ and $w:=\lceil(a+1)(b+1) / 2\rceil$. Assume for the moment to have proved Theorem 1 for the triple $(a, b, t)$ and fix any comb $L_{1} \cup \cdots \cup L_{t}$ solving it. For any integer $d<t$ the comb $L_{1} \cup \cdots \cup L_{d}$ is a solution of Theorem 1 for the triple $(a, b, d)$. Assume for the moment to have proved Theorem 1 for the triple $(a, b, w)$ and fix any comb $E$ solving it. For any integer $d>w$ any comb containing $E$ and intersecting transversally $Q$ 
is a solution of Theorem 1 for the triple $(a, b, d)$.

(ii) First assume that either $a=0$ or $b=0$. In this case it is sufficient to observe the existence of a degree $d$ comb $Y \subset \mathbb{P}^{3}$ intersecting transversally $Q$, with $L_{1}$ as its spine and such that no two of the $2 d$ points of $Y \cap Q$ are contained in a line of $Q$.

(iii) Assume $a=b=1$. If $d=1$, then we only need that $L_{1}$ is transversal to $Q$. We excluded the case $d=2$ and $a=b=1$. If $d \geq 3$ it is sufficient to take as $L_{i}, 2 \leq i \leq d$, general lines intersecting $L_{1}$, because if $L_{2}$ and $L_{3}$ are general, then the 6 points $Q \cap\left(L_{1} \cup L_{2} \cup L_{3}\right)$ are not contained in a plane.

(iii) Now assume $a=1$ and $b \geq 2$. Let $C \subset Q$ be a smooth curve of type $(1, b)$ containing $O^{\prime}$, but not containing $O$. We also assume that the tangent developable of $C$ does not contain $L_{1}$ (either take $C$ general in $\left|\mathcal{O}_{Q}(1, b)\right|$ or fix any $C$ through $O^{\prime}$ and then take as $L_{1}$ a general line through $O^{\prime}$ ). Fix $b$ general points $O_{1}, \ldots O_{b} \in L_{1}$. Since $O_{i} \notin Q$, for any line $T_{i}$ through $O_{i}$ Bezout theorem gives $\operatorname{deg}\left(T_{i} \cap C\right) \leq 2$. Since $b \geq 2$, the linear projection from $O_{i}$ does not induce an embedding of $C$ into a plane. Hence there is a line $R_{i} \subset \mathbb{P}^{3}$ such that $O_{i} \in R_{i}$ and $\operatorname{deg}\left(R_{i} \cap C\right) \geq 2$. We saw that $\operatorname{deg}\left(R_{i} \cap C\right) \leq 2$. Hence $\operatorname{deg}\left(R_{i} \cap C\right)=2$. Since $O_{i}$ is not contained in the tangential variety of $C$, the line $T_{i}$ is quasi-transversal to $C$ and it intersects $C$ at exactly two points. Take any $R_{1}$ as above and assume constructed $R_{1}, \ldots, R_{i}, 1 \leq i<b$, as above so that $E_{i}:=L_{1} \cup R_{1} \cup \cdots \cup R_{i}$ is a comb with spine $L_{1}$ and $\sharp\left(\left(R_{1} \cup \cdots R_{i}\right) \cap C\right)=2 i$. We take as $O_{i+1}$ a general point of $L_{1}$ so that $R_{i+1} \cap R_{j}=\emptyset$ for all $j<i$. In this way we construct a comb $E:=L_{1} \cup R_{1} \cup \cdots \cup R_{b}$ such that $\sharp\left(R_{i} \cap C\right)=2$ for all $i, \sharp(E \cap C)=2 b+1$ and $O \notin E$. Since $C \cong \mathbb{P}^{1}$ and $\operatorname{deg}\left(\mathcal{O}_{C}(1, b)\right)=2 b$, we have $h^{i}\left(C, \mathcal{I}_{E \cap C}(1, b)\right)=0, i=0,1$. Hence $h^{i}\left(Q, \mathcal{I}_{E \cap Q}(1, b)\right)=h^{0}\left(Q, \mathcal{I}_{O}\right)=0$, $i=1,2$. Hence Theorem 1 is true in this case.

(iv) Now assume $a=2$. By step (ii) we may assume $b>0$. Fix two lines $T_{i} \in\left|\mathcal{O}_{Q}(1,0)\right|, i=1,2$, such that $T_{1} \neq T_{2}$ and $L_{1} \cap\left(T_{1} \cup T_{2}\right)=\emptyset$. Any 3 disjoint lines of $\mathbb{P}^{3}$ are contained in a smooth quadric. Hence for general lines $L_{2}, \ldots, L_{b+1}$ meeting $L_{1}, T_{1}$ and $T_{2}$ the set $L_{1} \cup R_{1} \cup \cdots \cup R_{b+1}$ is a degree $b+2$ comb intersecting $Q$ in $O, O^{\prime}$ and $b+1$ points of each line $T_{i}$. For $b+2 \leq j \leq$ $d-1$, let $R_{j}$ be a general line intersecting $L_{1}$. Set $F:=L_{1} \cup R_{1} \cup \cdots \cup R_{d-1}$, $F^{\prime}:=R_{1} \cup \cdots \cup R_{b+1}$ and $F^{\prime \prime}:=L_{1} \cup R_{b+2} \cup \cdots \cup R_{d-1}$. Since $F^{\prime \prime} \cap T_{i}=\emptyset$ and $\operatorname{deg}\left(F \cap T_{i}\right)=b+1$, we have $h^{i}\left(Q, \mathcal{I}_{F \cap Q}(a, b)\right)=h^{i}\left(Q, \mathcal{I}_{F^{\prime \prime}}(0, b)\right), i=0,1$. Apply step (ii) for the pair $(0, b)$ to $F^{\prime \prime}$.

(v) Now assume $a \geq 3$. By steps (ii) and (iii) we may assume $b \geq 2$. We use induction on $a$. We adapt step (iv) in the following way. By step (i) we 
may assume $d \geq b+2$. Fix two lines $T_{i} \in\left|\mathcal{O}_{Q}(1,0)\right|, i=1,2$, such that $T_{1} \neq T_{2}$ and $L_{1} \cap\left(T_{1} \cup T_{2}\right)=\emptyset$. Any 3 disjoint lines of $\mathbb{P}^{3}$ are contained in a smooth quadric. Hence for general lines $L_{2}, \ldots, L_{b+1}$ meeting $L_{1}, T_{1}$ and $T_{2}$ the set $L_{1} \cup R_{1} \cup \cdots \cup R_{b+1}$ is a degree $b+1$ comb intersecting $Q$ in $O, O^{\prime}$ and $b+1$ points of each line $T_{i}$. Let $F^{\prime \prime} \subset \mathbb{P}^{3}$ be a general comb of degree $d-b-2$ with $L_{1}$ as its spine. By the inductive assumption we may assume $h^{0}\left(Q, \mathcal{I}_{F^{\prime \prime} \cap Q}(a-2, b)\right)=\max \{0,(a-1)(b+1)-2 d-2 b-2\}$ and $h^{1}\left(Q, \mathcal{I}_{F^{\prime \prime} \cap Q}(a-\right.$ $2, b))=\max \{0,2 d-2 b-2-(a-1)(b+1)\}$. Set $F:=F^{\prime \prime} \cup R_{1} \cup \cdots \cup R_{b+1}$. Since $F^{\prime \prime} \cap T_{i}=\emptyset$ and $\operatorname{deg}\left(F \cap T_{i}\right)=b+1$, we have $h^{i}\left(Q, \mathcal{I}_{F \cap Q}(a, b)\right)=h^{i}\left(Q, \mathcal{I}_{F^{\prime \prime}}(0, b)\right)$, $i=0,1$.

Lemma 1. Fix integers $r \geq 3, d>0$ and $t>0$. Let $Q \subset \mathbb{P}^{r}$ be a quadric hypersurface with rank 0, i.e. $Q=2 H$ with $H$ a hyperplane. Then there is a degree $d$ tree $Y \subset \mathbb{P}^{r}$ such that $Q$ contains no line of $Y, h^{0}\left(Q, \mathcal{I}_{Y \cap Q}(t)\right)=$ $\max \left\{0,\left(\begin{array}{c}r+t \\ r\end{array}\right)-\left(\begin{array}{c}r+t-2 \\ r\end{array}\right)-2 d\right\}$ and $h^{1}\left(Q, \mathcal{I}_{Y \cap Q}(t)\right)=\max \left\{0,2 d-\left(\begin{array}{c}r+t \\ r\end{array}\right)+\left(\begin{array}{c}r+t-2 \\ r\end{array}\right)\right\}$.

Proof. Since the case $t=1$ is trivial, we may assume $t \geq 2$. Set $u:=$ $\left\lfloor\left(\left(\begin{array}{c}r+t \\ r\end{array}\right)-\left(\begin{array}{c}r+t-2 \\ r\end{array}\right)-2 d\right) / 2\right\rfloor$ and $w:=\left\lceil\left(\left(\begin{array}{c}r+t \\ r\end{array}\right)-\left(\begin{array}{c}r+t-2 \\ r\end{array}\right)-2 d\right) / 2\right\rceil$. For any two distinct points $O, P$ of a projective space, let $\langle\{O, P\}\rangle$ denote the line spanned by $O$ and $P$.

(i) First assume $d \leq u$. In this case we need to find $Y$ such that

$$
h^{1}\left(Q, \mathcal{I}_{Q \cap Y}(t)\right)=0 .
$$

We use induction on $d$, starting from the obvious case $d=1$. Fix a tree $A \subset \mathbb{P}^{r}$ such that $Q$ contains no component of $A, \operatorname{deg}(A)=d-1$ and $h^{1}\left(Q, \mathcal{I}_{A \cap Q}(t)\right)=$ 0, i.e. $h^{0}\left(Q, \mathcal{I}_{A \cap Q}(t)\right)=\left(\begin{array}{c}r+t \\ r\end{array}\right)-\left(\begin{array}{c}r+t-2 \\ r\end{array}\right)-2 d-2$. Let $\tau$ be the type of $A$. Let $\mathcal{B}$ be the base scheme of the $\mathcal{O}_{Q}$-sheaf $\mathcal{I}_{A \cap Q}(t)$. By definition $\mathcal{B} \subset Q$ and $H^{0}\left(Q, \mathcal{I}_{\mathcal{B}}(t)\right)=H^{0}\left(Q, \mathcal{I}_{A \cap Q}(t)\right)$. Since $t \geq 2$ and the restriction map $\rho_{t}: H^{0}\left(\mathbb{P}^{r}, \mathcal{O}_{\mathbb{P}^{r}}(t)\right) \rightarrow H^{0}\left(Q, \mathcal{O}_{Q}(t)\right)$ is surjective, $\mathcal{B}$ is the base scheme of the $\mathcal{O}_{\mathbb{P}^{r} \text {-sheaf }} \mathcal{I}_{A \cap Q, \mathcal{O}_{\mathbb{P}}}(t)$. It is sufficient to find a line $L \subset \mathbb{P}^{r}$ such that $L \nsubseteq Q$, $A \cup L$ is a tree and $h^{0}\left(Q, \mathcal{I}_{(A \cup L) \cap Q}(t)\right)=\left(\begin{array}{c}r+t \\ r\end{array}\right)-\left(\begin{array}{c}r+t-2 \\ r\end{array}\right)-2 d$. Since $\rho_{t}$ is surjective and $(A \cup L) \cap Q \subset Q$, it is sufficient to prove $h^{0}\left(\mathbb{P}^{r}, \mathcal{I}_{(A \cup L) \cap Q, \mathcal{O} p}(t)\right)=$ $h^{0}\left(\mathbb{P}^{r}, \mathcal{I}_{A \cap Q, \mathcal{O}_{\mathrm{P}}}(t)\right)-2$.

(i1) First assume $H \subseteq \mathcal{B}$. Since no component of $A$ is contained in $H$, we get $h^{0}\left(\mathbb{P}^{r}, \mathcal{I}_{A \cap Q}(t)\right)=h^{0}\left(\mathcal{P}^{r}, \mathcal{I}_{A \cap H}(t-1)\right)$. Deforming $A$ to a general tree with type $\tau$ we may assume that $A \cap H$ is formed by $d-1$ general points of $H$. Hence $h^{0}\left(\mathbb{P}^{r}, \mathcal{I}_{A \cap H}(t-1)\right)=\left(\begin{array}{c}r+t-1 \\ r-1\end{array}\right)-\min \left\{\left(\begin{array}{c}r+t-2 \\ r-1\end{array}\right), d-1\right\}$. If $d-1 \leq\left(\begin{array}{c}r+t-2 \\ r-1\end{array}\right)$, then we get $h^{0}\left(\mathbb{P}^{r}, \mathcal{O}_{\mathbb{P}^{r}}(t)\right)-h^{0}\left(\mathbb{P}^{r}, \mathcal{I}_{A \cap Q}(t)\right)=\left(\begin{array}{c}r+t-1 \\ d-1\end{array}\right)+(d-1)>2(d-1)$, absurd. Now assume $d-1>\left(\begin{array}{c}r+t-2 \\ r-1\end{array}\right)$. In this case we get $h^{0}\left(\mathbb{P}^{r}, \mathcal{O}_{\mathbb{P}^{r}}(t)\right)-h^{0}\left(\mathbb{P}^{r}, \mathcal{I}_{A \cap Q}(t)\right)=$ 
$h^{0}\left(Q, \mathcal{O}_{Q}(t)\right)$, i.e. $h^{0}\left(Q, \mathcal{I}_{A \cap Q}(t)\right)=0$, contradicting the assumption $d-1<u$.

(i2) Now assume $H \nsubseteq \mathcal{B}$, but that the scheme $H \cap \mathcal{B}$ contains a degree $t$ hypersurface of $H$. Equivalently, assume that the restriction map $\rho_{H}$ : $H^{0}\left(Q, \mathcal{I}_{A \cap H}(t)\right) \rightarrow H^{0}\left(H, \mathcal{O}_{H}(t)\right)$ has rank 1 . The inequalities in step (i1) must be modified just by one. We get again a contradiction. Hence in step (i4) we are allowed to assume that $\rho_{H}$ has rank $\geq 2$.

(i3) Assume $d \leq r$. First assume $r=3$. Let $2 L \subset \mathbb{P}^{2}$ be a double line. Let $E=L_{1} \cup L_{2} \subset \mathbb{P}^{2}$ be a reducible conic such that $L \nsubseteq E$. The degree 4 scheme $2 L \cap E$ satisfies $h^{0}\left(\mathbb{P}^{2}, \mathcal{I}_{E \cap 2 L}(2)\right)=2$. Hence $h^{1}\left(\mathbb{P}^{2}, \mathcal{I}_{E \cap 2 L}(2)\right)=0$. Hence $h^{1}\left(2 L, \mathcal{I}_{E \cap 2 L}(2)\right)=0$. See $2 L$ as a hyperplane section $N \cap Q$ of $Q$. Notice that $E \cap 2 L$ is the base locus of the linear system $\left|\mathcal{I}_{E \cap Q}(2)\right|$. Hence for any $P \in Q \backslash 2 L$ we have $h^{0}\left(\mathbb{P}^{3}, \mathcal{I}_{(E \cap 2 L) \cup\{P\}}(2)\right)=5$. Fix a general quadric surface $Q_{1}$ containing $(E \cap L) \cup\{P\}$. Since $h^{0}\left(\mathbb{P}^{2}, \mathcal{I}_{E \cap 2 L}(2)\right)=2, Q_{1}$ is not a cone with vertex containing $P$. Hence there is $i \in\{1,2\}$ and $O \in L_{i} \backslash L_{1} \cap L_{2}$ such that that there is a quadric surface $Q_{1}$ containing $\{P\} \cup(E \cap 2 L)$, but not the tangent vector $w$ of $\langle\{O, P\}\rangle$ at $P$. Hence $h^{1}\left(\mathbb{P}^{3}, \mathcal{I}_{(E \cap 2 L) \cup w}(2)\right)=0$. Since $(2 L \cap E) \cup w \subset Q$, we get $h^{1}\left(Q, \mathcal{I}_{(2 L \cap E) \cup w}(2)\right)=0$. Since $t \geq 2$ we have $h^{1}\left(Q, \mathcal{I}_{(2 L \cap E) \cup w}(2)\right)=0$. Since $E \cup\langle\{O, P\}\rangle$ is a tree, we conclude the case $r=3$ and $d \leq 3$. Now assume $r \geq 4$. We use induction on $r$. Let $M \subset \mathbb{P}^{r}$ be a general hyperplane. Set $Q^{\prime}:=Q \cap M$. Notice that $Q^{\prime}$ is the double of the hyperplane $H \cap M$ of $M$. Let $\rho^{\prime}: H^{0}\left(Q, \mathcal{O}_{Q}(2)\right) \rightarrow H^{0}\left(Q, \mathcal{O}_{Q}(2)\right)$ be the restriction map. If $d<r$ we take a degree $d$ tree $B \subset M$ such that $h^{1}\left(Q^{\prime}, \mathcal{I}_{Q^{\prime} \cap B}(2)\right)=0$. Hence $h^{1}\left(Q, \mathcal{I}_{Q \cap B}(2)\right)=0$. Since $t \geq 2$, we get $h^{1}\left(Q, \mathcal{I}_{Q \cap B}(t)\right)=0$. Now assume $d=r$. The inductive assumption give the existence of a degree $d-1$ tree $A \subset M$ such that $h^{1}\left(Q^{\prime}, \mathcal{I}_{A \cap Q^{\prime}}(2)\right)=0$. Let $L \subset \mathbb{P}^{r}$ be a line such that $L \nsubseteq M$ and $L$ contains a smooth point of $A$. Notice that $A \cup L$ is a tree. Since the restriction map is surjective and $t \geq 2$, it is sufficient to find $L$ such that $h^{0}\left(\mathbb{P}^{r}, \mathcal{I}_{\left(A \cup Q^{\prime}\right) \cup L \cap Q}(2)\right)=h^{0}\left(\mathbb{P}^{r}, \mathcal{I}_{\left(A \cup Q^{\prime}\right)}(2)\right)-2$. This is done as in the case $r=3$, because $A$ spans $M$.

(i4) Assume $d \geq r+1$. Fix a general $f \in H^{0}\left(\mathbb{P}^{r}, \mathcal{I}_{A \cap Q}(t)\right)$ and set $U:=\{f=$ 0 \}. Bertini's theorem says that $U$ is smooth outside $\mathcal{B}$. By step (i1) $U$ does not contains (i2). By step (i2) $U \cap H$ is not contained in $\mathcal{B} \cap H$. Hence there is $P \in$ $U \cap H$ such that $P$ is smooth at $U$. Since $d-1 \geq r$ and we may take as $A$ a general tree of type $\tau$, the tree $A$ spans $\mathbb{P}^{r}$. Since $A$ spans $\mathbb{P}^{r}$ there is $S \subset A_{\text {reg }}$ such that $\sharp(S)=r$, the tangent vectors at $P$ of the lines $\langle\{O, P\}\rangle, O \in S$, are linearly independent and for each $O \in S A \cup\langle\{O, P\}\rangle$ is a tree (notice that for some $f$ we may take as $P$ a general point of $H$ ). Since $U$ is smooth at $P$, we may find 
$O \in A_{\text {reg }}$ such that $A \cup\langle\{O, P\}\rangle$ is a tree and the tangent vector $w$ of $\langle\{O, P\}\rangle$ at $P$ is not contained in $U$. Hence $f \notin H^{0}\left(\mathbb{P}^{r}, \mathcal{I}_{(A \cap Q) \cup w}(t)\right)$. Since $P \notin \mathcal{B}$, we have $h^{0}\left(\mathbb{P}^{r}, \mathcal{I}_{(A \cap Q) \cup\{P\}}(t)\right)=h^{0}\left(\mathbb{P}^{r}, \mathcal{I}_{A \cap Q}(t)\right)-1$. Since $f \in H^{0}\left(\mathbb{P}^{r}, \mathcal{I}_{(A \cap Q) \cup\{P\}}(t)\right)$, while $f \notin H^{0}\left(\mathbb{P}^{r}, \mathcal{I}_{(A \cap Q) \cup w}(t)\right)$ and $f \notin H^{0}\left(\mathbb{P}^{r}, \mathcal{I}_{(A \cap Q) \cup w}(t)\right)$, we again obtain $h^{0}\left(\mathbb{P}^{r}, \mathcal{I}_{(A \cap Q) \cup w}(t)\right)=h^{0}\left(\mathbb{P}^{r}, \mathcal{I}_{A \cap Q}(t)\right)-2$. Hence $h^{1}\left(\mathbb{P}^{r}, \mathcal{I}_{(A \cap Q) \cup w}(t)\right)=0$.

(ii) Now assume $d>u$. In this range we need to find $Y$ with $h^{0}\left(Q, \mathcal{I}_{Y \cap Q}(t)\right)=$ 0 . As in step (i) of the proof of Theorem 1 to do all cases with $d>u$ it is sufficient to do the case $d=w$. If $w=u$, then we just proved this case in step (i). Now assume $w=u+1$, i.e. assume that $\left(\begin{array}{c}r+t \\ r\end{array}\right)-\left(\begin{array}{c}r+t-2 \\ r\end{array}\right)$ is an odd integer. Let $A \subset \mathbb{P}^{r}$ be a degree $u$ tree such that $h^{1}\left(Q, \mathcal{I}_{A \cap Q}(t)\right)=0$, i.e. $h^{0}\left(Q, \mathcal{I}_{A \cap Q}(t)\right)=1$. First assume that $H$ is not contained in the base locus $\mathcal{B}$ of $\mathcal{I}_{A \cap Q}(t)$. We may take as degree $d$ tree any tree $A \cup L$ with $L$ containing a general point of $H$. Now assume $H \subseteq \mathcal{B}$. For general $A$ we have $h^{0}\left(\mathbb{P}^{r}, \mathcal{I}_{A \cap Q}(t)\right)=h^{0}\left(\mathbb{P}^{r}, \mathcal{I}_{A \cap H}(t-1)=\left(\begin{array}{c}r+t-1 \\ r\end{array}\right)-u<h^{0}\left(\mathbb{P}^{r}, \mathcal{I}_{Q} t\right)\right)$, a contradiction.

Proof of Theorem 2. Any two quadric hypersurfaces of $\mathbb{P}^{r}$ with the same rank are projectively equivalent. Hence Theorem 2 is true for one rank $e$ hyperquadric if and only if it is true for all rank $e$ hyperquadrics. Lemma 1 proves the case $e=0$. Now assume $e>0$. There is a flat family of rank $e$ hyperquadrics of $\mathbb{P}^{r}$ with as a flat limit a double hyperplane $2 H$. Apply Lemma 1 to $2 H$ and then use the semicontinuity theorem for cohomology.

\section{Intersection with a Hyperplane}

Let $M \subseteq \mathbb{P}^{r}$ be a reduced variety. A tangent vector of $M$ is a degree 2 connected zero-dimensional scheme $Z \subset M_{\text {reg }}$. Fix a type $\tau:\{2, \ldots, d-1\} \rightarrow\{1, \ldots, d-$ $1\}$. For each $i \in\{1, \ldots, d\}$ the function $\tau \mid\{1, \ldots, i\}$ is a type for degree $i$ trees.

Remark 1. Let $H \subset \mathbb{P}^{r}$ be a hyperplane and $Y \subset \mathbb{P}^{r}$ any tree. If no line of $Y$ is contained in $H$, then the scheme $Y \cap H$ is the disjoint union of finitely many points and tangent vectors.

Any tree of degree $d$ has $d-1$ singular points. Fix $Y=L_{1} \cup \cdots \cup L_{d} \in$ $Z(r, d, \tau)$. For each $P \in \operatorname{Sing}(Y)$ let $\tau(P)$ be the two indices $i, j \in\{1, \ldots, d\}$ such that $\{P\}=L_{i} \cap L_{j}$. For each line $L \subseteq Y$ set $\sigma(L):=\sharp(S \cap L)$. Set $\sigma(i)=\sigma\left(L_{i}\right)$. The function $\sigma$ is uniquely determined by $\tau$. A final line of $Y$ is a line $L$ with $\sigma(L) \leq 1$ (and hence $\sigma(L)=1$ if $d \geq 2$ ). Any bamboo of degree $d \geq 2$ has exactly two ordering of its components associated to the type of any bamboo. $Y$ is comb $\Longrightarrow$ there is a line $L \subseteq Y$ such that $\sigma(L)=d-1 \Longrightarrow$ 
$\sigma(1)=d-1$. A comb has a unique type. Assume $d \geq 4$. The wire $w(Y)$ of $Y$ is the maximal cardinality of a set $S^{\prime} \subseteq \operatorname{Sing}(Y)$ such that the linear space $\left\langle S^{\prime}\right\rangle$ spanned by $S^{\prime}$ contains no line of $Y$. Obviously $w(Y) \leq \min \{d-1, r\}$. The wire $w(\tau)$ is the integer $w(Y)$, where $Y$ is a general element of $Z(r, d, \tau)$. For many $\tau$ there are $Y_{1}, Y_{2} \in Z(r, d, \tau)$ such that $w\left(Y_{1}\right)<w(\tau)<w\left(Y_{2}\right)$.

Remark 2. For any tree $Y \subset \mathbb{P}^{r}$ the wire $w(Y)$ is the only integer with the following properties:

(i) There is a hyperplane of $\mathbb{P}^{r}$ containing no line of $Y$ and containing exactly $w(Y)$ singular points of $Y$.

(ii) Every hyperplane of $\mathbb{P}^{r}$ containing at least $w(Y)+1$ singular points of $Y$ contains a component of $Y$.

Proposition 1. Fix an integer $d>0$, a hyperplane $H \subset \mathbb{P}^{r}$ and a set $S \subset H$ such that $\sharp(S)=d$ and no 3 of the points of $S$ are collinear. Fix any type $\tau$ for trees of degree $d$. Then there is $Y \in Z(r, d, \tau)$ such that $Y \cap H=S$.

Proof. Take an ordering $P_{1}, \ldots, P_{d}$ of the points of $S$. Let $L_{1}$ be a general line through $P_{1}$. Hence we may assume $d \geq 2$ and use induction on $d$. Take $A \in Z(r, d-1, \tau \mid\{1, \ldots, d-1\})$ such that $A \cap H=\left\{P_{1}, \ldots, P_{d-1}\right\}$. Let $E$ be the plane spanned by $L_{\tau(d)}$ and by $P_{d}$. We claim that $L_{\tau(d)}$ is the only line of $A$ contained in $E$. Assume that this is not true and call $B$ another line of $A \cap E$. The set $\left\{P_{d}\right\} \cup\left(L_{\tau(d)} \cup B\right) \cap H$ contains 3 collinear points, contradicting our assumption. Hence we may take $Y=A \cup L_{d}$ with $L$ a general line through $P_{d}$ contained in the plane $E$.

Proposition 2. Fix a hyperplane $H \subset \mathbb{P}^{r}, r \geq 3$, a finite set $S \subset H$ and $P \in S$. Then there is a comb $Y \subset \mathbb{P}^{r}$ such that $Y \cap H=S$ and $P$ is the intersection of $H$ with the spine of $Y$ if and only if $\sharp(D \cap S) \leq 2$ for each line $D \subset H$ containing $P$.

Proof. First assume $\sharp(D \cap S) \leq 2$ for each line $D \subset H$ containing $P$. Take an ordering $P_{1}, \ldots, P_{d}$ of the points of $S$ with $P_{1}:=P$. Let $L_{1}$ be a general line through $P_{1}$. Hence we may assume $d \geq 2$ and use induction on $d$. Take a degree $d-1$ comb $A$ with $L_{1}$ as its such that $A \cap H=\left\{P_{1}, \ldots, P_{d-1}\right\}$. The plane $B$ spanned by $L_{1}$ and $P_{d}$ contain no points of $A \backslash L$, because $T \cap L_{1} \neq \emptyset$ the line $D$ through $P_{1}$ and $P_{2}$ would contain the point $T \cap H$.

Remark 3. Fix an integer $d \geq 2$. Let $Y \subset \mathbb{P}^{r}$ be a tree. The following conditions are equivalent:

1. $Y$ is a bamboo; 
2. $Y$ has only two final lines;

3. no line of $Y$ intersect at least two other components of $Y$.

Proposition 3. Assume $d$ even. Let $H \subset \mathbb{P}^{r}$ be a hyperplane. Let $Z \subset H$ be a disjoint union of $d / 2$ tangent vectors. Assume $\operatorname{deg}(D \cap Z) \leq 2$ for all lines $D \subset H$. Then there is a tree $Y \subset \mathbb{P}^{r}$ such that $Y \cap H=Z$. Any such tree is a bamboo.

Proof. We use induction on the integer $d / 2$, the case $d=2$ being obvious. Take any $P \in Z_{\text {red }}$. Call $v$ the connected component of $Z$ with $P$ as its reduction and set $Z^{\prime}:=Z \backslash v$. Let $A=L_{1} \cup \cdots \cup L_{d / 2-1} \subset \mathbb{P}^{r}$ be a bamboo such that $Z^{\prime}=Z \cap B$. Let $B$ be the plane spanned by $L_{d / 2-1}$ and $P$. We claim that there is no line $L \subset A$ such that $L \neq L_{d / 2-1}$ and $L \subset B$. Assume the existence of $L$. The set $H \cap B$ is the line spanned by $L_{d / 2-1} \cap H$ and $P$. Since $L \cap H \subset H \cap B$, we get $\operatorname{deg}(D \cap Z) \geq 3$, a contradiction. Fix a general plane $U \subset \mathbb{P}^{r}$ containing $R$. Since $v \subset R$, we have $R \cap A=\emptyset$. Hence $U \cap A$ is finite. Hence we may take as $L_{d}$ a general line in $U$ containing $P$.

Part 3 of Remark 3 implies that any such tree is a bamboo.

Theorem 3. Fix integers $d \geq 2 a \geq 0, d>0$. Let $H \subset \mathbb{P}^{r}$ be a hyperplane. Let $Z \subset H$ be a disjoint union of a tangent vectors and $d-2 a$ points. Assume $\operatorname{deg}(D \cap Z) \leq 2$ for all lines $D \subset H$. Then there is a degree $d$ bamboo $Y \subset \mathbb{P}^{r}$ such that $Y \cap H=Z$.

Proof. If $d=2 a$, then apply Proposition 3. Now assume $d>2 a$. Take $Z=S \sqcup W$ with $S$ union of the reduced connect components of $Z$ and $W$ the union of the tangent vectors. First apply the proof of Proposition 1 to $S$ (say obtaining a bamboo $L_{1} \cup \cdots L_{d-2 a}$ and then apply the proof of Proposition 3 to $W$, starting from any $P \in W_{\text {red }}$ and taking as $L_{d-2 a+1}$ a general line through $P$ intersecting $L_{d-2 a+1}$.

Remark 4. Fix an odd integer $d \geq 3$ and a hyperplane $H \subset \mathbb{P}^{r}$. Let $Z \subset H$ be a disjoint union of $(d-1) / 2$ tangent vector and one point $P$. Assume the existence of a degree $d$ tree $Y \subset \mathbb{P}^{r}$ such that $Z=H \cap Y$ and call $L$ the line of $Y$ containing $P$. Then the projective curve $\overline{Y \backslash L} \subset \mathbb{P}^{r}$ is a disjoint union of bamboos of even degree. 


\section{Acknowledgements}

The author was partially supported by MIUR and GNSAGA of INdAM (Italy).

\section{References}

[1] E. Ballico, Ph. Ellia, Generic curves of small genus in $\mathbb{P}^{3}$ are of maximal rank, Math. Ann., 264 (1983), 211-225. 
